\title{
Existence of four-dimensional polymer collapse I. Kinetic growth trails
}

\author{
A.L. Owczarek ${ }^{\mathrm{a}, *}$, T. Prellberg ${ }^{\mathrm{b}}$ \\ a Department of Mathematics and Statistics, The University of Melbourne, Parkville, \\ Victoria 3052, Australia \\ b Department of Theoretical Physics, University of Manchester, Manchester M13 9PL, UK \\ Received 14 March 1998
}

\begin{abstract}
We present the results of simulations of kinetic growth trails (KGT) (bond-avoiding walks) in four dimensions. We use a mapping from a kinetic growth model to a static model of selfinteracting trails (ISAT) at a particular temperature to argue that this temperature is precisely the collapse temperature of four-dimensional interacting trails. To do this we show that the kinetic growth trails behave neither like static non-interacting trails, which should behave as excludedvolume-dominated four-dimensional polymers (that is self-avoiding walks), or collapsed fourdimensional polymers, but rather show an intermediate behaviour. This is the first indication of collapse in any four-dimensional lattice polymer model and so may be helpful in deciding which of the competing models of polymers is a good model in lower dimensions. We have calculated various exponents of the KGT model and identified them with certain critical exponents of the static ISAT problem. (C) 1998 Elsevier Science B.V. All rights reserved.
\end{abstract}

PACS: 05.50.+q; 61.41.+e; 05.70.Fh

Keywords: Trails; Self-interacting trails; Kinetic growth; Lattice polymers; Polymer collapse

\section{Introduction}

There has been continuing interest in statistical mechanical models of the conformations of linear polymers in dilute solutions in two and three dimensions [1]. One aim of these studies is to extract the universal (critical) scaling properties of these models, since they should hold exactly for a range of physical systems. It is expected that the models display three distinct behaviours. At high temperatures, they should mimic polymers in a 'good' solvent, where the excluded volume effect is dominant. Opposing this, at low temperatures the model should describe a collapsed, internally dense, state caused by the attractive interactions of different sections of a polymer

\footnotetext{
*E-mail: aleks@ms.unimelb.edu.au.
} 
mediated by a solvent. At one temperature, known as the $\theta$-point, the excluded volume and attractive forces 'balance' to give a third, intermediate behaviour. This third behaviour is often approximated by a simple random walk model. However, it was realised some time ago that in low dimensions the real situation is more complex. In two dimensions, the $\theta$-state does not share the scaling features of a random walk $[2,3]$. In three dimensions the two equivalent descriptions of the $\theta$-state are given by the continuum Edwards model with two and three body forces [4,5] and the $n \rightarrow 0$ limit of the magnetic (tricritical) $\left(\phi^{2}\right)^{2}-\left(\phi^{2}\right)^{3} \mathrm{O}(n)$ field theory [6-8]. The analysis of these theories is subtle and predicts that $d=3$ is the upper critical dimension of the $\theta$-state, and therefore the critical behaviour of this state is given by mean-field (Flory) theory predictions modified by logarithmic factors. On the other hand, the canonical model of polymer collapse in lattice statistical mechanics has been self-avoiding walks (SAW) on a regular lattice interacting via nearest-neighbour attraction, or ISAW. This model manifestly includes the 'excluded volume' condition of real polymers as well as the attraction necessary for collapse. There has not been a direct demonstration that the behaviour of this model around its collapse point is given by the Edwards model, although it is widely accepted as such [9].

Little consideration has been given to four-dimensional polymer collapse. However, several points make it worthwhile for study, especially as it points to the correct description of the lower-dimensional systems. As far as we are aware there is no clear understanding of the Edwards model with respect to collapse in four dimensions. This is the upper critical dimension for the excluded volume problem: the two parameter Edwards model predicts that excluded volume dominated polymers should scale as a random walk modified by multiplicative logarithmic factors. The two parameter Edwards model also implies that a crossover to a collapse state, if it exists, has crossover exponent 0 . This presumably implies via scaling arguments [10] a specific heat exponent of $\alpha=-\infty$ which is difficult to interpret. In fact there is a long standing conjecture [11] that the collapse transition disappears in four dimensions, at least at finite temperature. On the other hand, given that the collapse transition has an upper critical dimension of three, one might expect from experience with other critical phenomena, such as the ferromagnetic Ising model, that the collapse transition exists but does so with some sort of mean field behaviour. In this case we assume the mean field behaviour to imply a pure random walk state for the $\theta$-state. Whether the associated thermodynamic transition would give a simple jump in the specific heat or some other simple singularity varying with dimension is unclear. To look at these questions we have embarked on several studies. The first of these we report here.

Another lattice model of polymer configurations studied extensively is that of selfavoiding trails (SAT) or trails for short [12-15]. These are paths on a lattice which have no two steps on the same bond of that lattice but may occupy the same site. This restriction is sometimes referred to as bond-avoiding, in contrast to self-avoiding walks (SAW) which are site avoiding (that is, no two vertices of the walk may occupy the same site on the lattice). Clearly walks are, by default, also bond-avoiding. Trails possess an excluded volume effect and it is believed that trails and SAW are in the same 
universality class $[16,17]$ which describes good solvent polymers. (For more recent discussion of the subtle differences between walks and trails regarding corrections to scaling see [18].) It has been shown [13] that there should exist a collapse transition when contact attraction is added to the trail model. Moreover, Shapir and Oono [13] have argued that this point should be tricritical in nature, as it is at the ISAW collapse point. However, they predict that ISAW and ISAT are in different universality classes. Importantly, while the upper critical dimension for ISAW is expected to be $d_{u}=3$, the Shapir-Oono field theory gives $d_{u}=4$ for ISAT. Therefore, this implies generically that logarithmic corrections occur at the $\theta$-point in four dimensions, which presumably should occur at a finite temperature.

On the other hand, three other scenarios have since been suggested for ISAT, deduced from the results of different Monte Carlo simulation techniques. Firstly, some Monte Carlo work [19] has suggested that ISAT and ISAW are in fact in the same universality class, so collapse in ISAT would then have an upper critical dimension of three. The behaviour in four dimensions is unknown for ISAW so no inference can be made about ISAT. A second scenario is that the upper critical dimension for ISAT is three but that the universality class is different to that of ISAW. This scenario would give no prediction of the type of behaviour expected at the collapse point by ISAT. Support for this scenario comes from studies of kinetic growth trails (KGT) [20,21], which are dynamic versions of these lattice paths that can be mapped onto the static problem at one particular temperature. In each case, the simulations of kinetic growth trails demonstrate the behaviour intermediate between the excluded volume state (as in SAW) and the collapsed one (which has fractal dimension $1 / d$ for $d$ dimensions). This has been used to argue that the temperature of the mapping is also the collapse temperature. Later [22] it was suggested that the exponents derived from the kinetic trail simulations were not the correct exponents for the ISAT collapse transition even though the temperature of the mapping is the collapse value: this is a subtle argument based on renormalisation considerations of the Edwards model. It was also suggested [22] from numerical work that the transition for ISAT in three dimensions is, in fact, first order rather than second order. Presumably, the same arguments could be used to imply the same situation in four dimensions. This gives us a third possible scenario.

One caveat in all of the above on ISAT is that we are assuming there is only one transition in the ISAT model. Evidence strongly suggests that this is the case for ISAW [23] and no other transition has been suggested from previous numerical work on ISAT $[14,19]$ but early workers on polymer collapse sometimes suggested that two transitions may take place, and recent work [24] on ISAW suggest that a second nonthermodynamic transition may take place in two dimensions. We shall not comment further on this possibility as the simulations presented here do not allow this.

So we have at least four different theoretic scenarios for ISAT. Firstly, the ShapirOono field theory where ISAT have an upper critical dimension of four. Secondly, that ISAW and ISAT have the same scaling behaviour at any temperature and thirdly that the upper critical dimension of ISAT is three but its collapse critical behaviour is different from that in ISAW in dimensions two and three. Finally, it is suggested that 
transitions in ISAT models (described by the kinetic growth trail algorithm) are firstorder ones [22]. Note that this last suggestion would invalidate the exponents inferred for the ISAT model from the kinetic growth simulations.

Now given that in four dimensions little is known about ISAW, ISAT or KGT we first thought it appropriate to discover the behaviour of KGT in four dimensions and see what we can infer about ISAT. Hence we have simulated kinetic growth trails in four dimensions. This allows us to find a collapse temperature in the ISAT model and predict that the $\theta$-state for ISAT in four dimensions is Gaussian. In further work we shall simulate ISAT and ISAW in four dimensions with standard Monte Carlo algorithms.

\section{Models}

We define the ISAT model on the four-dimensional hypercubic lattice in the following way. The lattice has coordination number 8 and we consider configurations $\varphi_{N}$ of trails, or bond-avoiding walks, of length $N$ starting from a fixed origin. Let $m_{k}, k=1, \ldots, 4$ be the number of sites of the lattice that has been visited $k$ times by the trail. The partition function of a very general interacting trail model is

$$
Z_{N}\left(\omega_{2}, \omega_{3}, \omega_{4}\right)=\sum_{\varphi_{N}} \omega_{2}^{m_{2}} \omega_{3}^{m_{3}} \omega_{4}^{m_{4}}
$$

where $\omega_{k}$ is the Boltzmann weight associated with $k$-visited sites. The canonical model is one where every segment of the trail at some contact site interacts with every other segment at that site, so that

$$
\omega_{k}=\omega^{\left(\begin{array}{c}
k \\
2
\end{array}\right)} \text { for } k=2,3,4,
$$

with $\omega \equiv \omega_{2}$. Now, two other reasonably natural models are defined by

$$
\omega_{k}=\omega^{k-1} \quad \text { for } k=2,3,4
$$

and

$$
\omega_{k}=\omega \quad \text { for } k=2,3,4 \text {. }
$$

It would be expected that each model possesses a collapse transition at some value of $\omega$, and that the transitions fall into the same universality class. Of course, if rather the coordination number of the lattice had been 4 then the only possibility is a single contact and all three models are isomorphic. The canonical model has been investigated in two and three dimensions using exact enumeration [14,25,15] and Monte Carlo techniques [26-28,19]. In two dimensions, recent work [20] provides a body of evidence that reinforces the hypothesis that ISAT and ISAW are truly in different universality classes. This work was accomplished using a kinetic growth algorithm. 
The growth model of kinetic trails was introduced by Lyklema [29] for reasons similar to those that led to the introduction of kinetic walks. It is defined on a fourdimensional hypercubic lattice as follows: choose a starting site on the lattice and dynamically construct a trail by adding steps at integer time intervals, choosing at each time step equally from the available nearest-neighbour sites that do not violate the bondavoiding condition. This produces trails of any length, with a particular distribution different to that of non-interacting (static) trails. A trail can visit a particular site up to four times. Except for the first step, if the kinetic trail is on a site that has not visited previously, there are seven choices (coordination number 8 lattice) of the next step and the probability of each is simply $\frac{1}{7}$. If the trail has previously visited the site $j$ (which can be 1,2 or 3 ) times, the choice of next step is restricted to $7-2 j$ possibilities and the probability of that step taken is $1 /(7-2 j)$. If one associates the step probabilities with the site from which it emanates then the associated probability of a site that has been visited $k$ times in total is

$$
p_{k}=\frac{1}{7 \cdot 5 \cdot \cdots \cdot(9-2 k)} \quad \text { for } k=1,2,3,4 .
$$

A kinetic trail on this lattice can hence be mapped onto a static ISAT model with fixed weights $\omega_{k}=7^{k} p_{k}$, whence

$$
\omega_{2}=\frac{7}{5}, \quad \omega_{3}=\frac{49}{15}, \quad \omega_{4}=\frac{343}{15} .
$$

Note that every static open trail configuration occurs as a kinetic trail. On the other hand, it is possible for kinetic trails to revisit the origin three times and so form a four-dimensional 'loop'. These loops are discarded from the kinetic algorithm after they form. We show later that in fact, as in three dimensions but unlike two dimensions, a finite proportion of the kinetic growth trails stay open no matter how long one grows them. This implies that the connective constant of the partition function of the static problem at this set of weights is $\mu=7$.

\section{Simulations and analysis techniques}

We have generated kinetic growth trails of various lengths up to $2^{16}$ steps. The occupied sites of the trail were stored by means of a hash table [30], with the hash index being computed from the coordinates. This enables efficient testing of self-avoidance without having to store the whole lattice, so that the generation of a walk of length $N$ requires time $O(N)$ only. The size of the hash table needs to exceed the maximal walk length only slightly, so that the memory requirement is also $O(N)$. When a walk reaches the desired maximal length or gets trapped, a new one gets generated, thereby ensuring the statistical independence of the walks sampled at fixed length.

We have calculated the proportion left open at various stages $P_{N}$, the root-meansquare end-to-end distances $R_{N}$, and the ratio $A_{N}$ of the fourth moment to the square of the end-to-end distances and information on the number of contacts for the calculation of the internal energy $U_{N}$ and the specific heat $C_{N}$ with estimates of statistical 
Table 1

Table of raw data I

\begin{tabular}{rllllll}
\hline \multicolumn{1}{l}{$N$} & $P_{N}$ & $\delta P_{N}$ & $U_{N}$ & $\delta U_{N}$ & $C_{N}$ & $\delta C_{N}$ \\
\hline 512 & 0.99329477 & 0.000025721 & 0.0130715437 & 0.0000012118 & 0.00751909 & 0.00000389 \\
1024 & 0.99321554 & 0.000025870 & 0.0133482172 & 0.0000008780 & 0.00789431 & 0.00000385 \\
2048 & 0.99318546 & 0.000025927 & 0.0135047162 & 0.0000006309 & 0.00815235 & 0.00000383 \\
4096 & 0.99327182 & 0.000040737 & 0.0135946853 & 0.0000007121 & 0.00830802 & 0.00000604 \\
8192 & 0.99323631 & 0.000040843 & 0.0136428697 & 0.0000005061 & 0.00839417 & 0.00000602 \\
16384 & 0.99318526 & 0.000040994 & 0.0136690690 & 0.0000003591 & 0.00845215 & 0.00000602 \\
32768 & 0.99325711 & 0.000066595 & 0.0136839184 & 0.0000004152 & 0.00847338 & 0.00000981 \\
65536 & 0.99316701 & 0.000105987 & 0.0136913748 & 0.0000004647 & 0.00848987 & 0.00001550
\end{tabular}

Table 2

Table of raw data II

\begin{tabular}{rllll}
\hline \multicolumn{1}{r}{$N$} & \multicolumn{1}{c}{$R_{N}$} & $\delta R_{N}$ & $A_{N}$ & $\delta A_{N}$ \\
\hline 512 & 26.286900 & 0.002927 & 1.496073 & 0.000980 \\
1024 & 37.186147 & 0.004148 & 1.497788 & 0.000983 \\
2048 & 52.605768 & 0.005874 & 1.498731 & 0.000985 \\
4096 & 74.392461 & 0.013144 & 1.499451 & 0.001560 \\
8192 & 105.193926 & 0.018590 & 1.499698 & 0.001560 \\
16384 & 148.766555 & 0.026309 & 1.500396 & 0.001563 \\
32768 & 210.368791 & 0.060654 & 1.498771 & 0.002542 \\
65536 & 297.544818 & 0.136080 & 1.501988 & 0.004047 \\
\hline
\end{tabular}

errors. To be extra careful we did independent simulations for each $N$ we collected data for. While this is not strictly necessary and time consuming it dismisses any possibility of correlated data in these type of variable length Monte Carlo simulations. We simulated a fixed number of lengths $N$ exponentially spaced. The raw data are given in Tables 1 and 2.

We simulated the model on an unbounded lattice to investigate the bulk behaviour. The simulations took approximately 10 CPU days on an Dec Alphastation 250/4/266. We have simulated $1 \times 10^{7}$ independent samples of lengths 512,1024 , and $2048,4 \times 10^{6}$ independent samples of lengths 8192 and 16384 and $1.5 \times 10^{6}$ independent samples of length $N=32768$ and finally $6 \times 10^{5}$ independent samples of length $N=65536$.

\section{Results and analysis}

We now present the analysis of the simulations of the kinetic trails. These simulations were carried out on the simple hypercubic lattice (4d-hc).

Our most important and convincing pieces of evidence arise from the study of the distribution of the end-to-end distances of our trails. We considered the behaviour of the mean-squared end-to-end distance $R_{N}^{2}=\left\langle R_{e}^{2}\right\rangle_{N}$. We first recall that in four dimensions in the swollen phase one has $R_{N}^{2} \sim R_{S} N(\log N)^{1 / 4}$ [31], whereas in the collapsed phase one expects $R_{N}^{2} \sim R_{c} N^{1 / 2}$. Attempting to fit the raw data to a power-law $N^{2 v}$ 


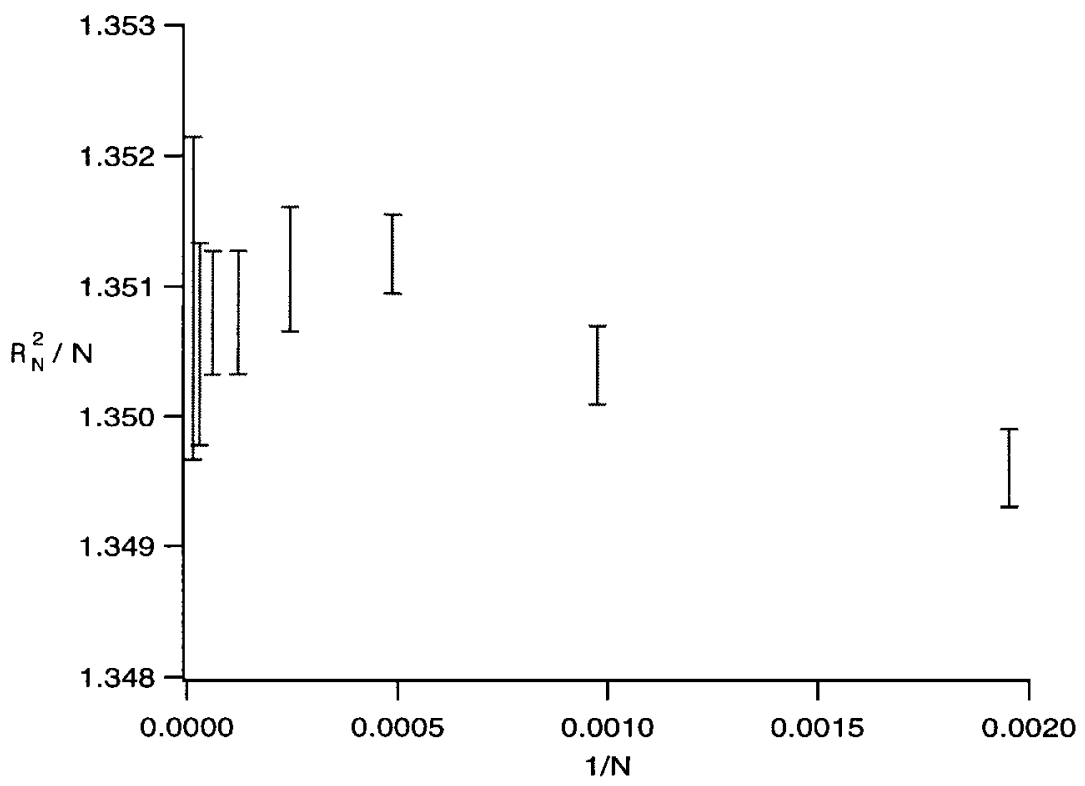

Fig. 1. $R_{N}^{2} / N$ is plotted against $1 / N$. Here, as in the next pictures, the error bars, which represent single standard deviations, are indicated explicitly.

gives an exponent close to 1 , that is $2 v=1.0002(2)$. Hence we have considered $R_{N}^{2} / N$. We have plotted $R_{N}^{2} / N$ versus $1 / N$ in Fig. 1 . We have used this default scale $1 / N$ for convenience as the values of $R_{N}^{2} / N$ are essentially constant for $N>2000$ within our error estimates. One sees that the ratio $R_{N}^{2} / N$ approaches $1.351(1)$ as $N \rightarrow \infty$. We conclude that

$$
v_{K G T}=\frac{1}{2} .
$$

Given that the factor $(\log N)^{1 / 4}$ is only a weak multiplier, that is difficult to rule out completely, we have also calculated the amplitude ratio

$$
A_{N}=\frac{\left\langle R_{\mathrm{e}}^{4}\right\rangle_{N}}{\left\langle R_{\mathrm{e}}^{2}\right\rangle_{N}^{2}}
$$

which we estimate approaches the value $A_{\infty}=1.500(2)$. The value for a random walk for this universal amplitude ratio is $\frac{3}{2}$. We conclude that our kinetic growth trails also approach $\frac{3}{2}$. Hence, given that $v=\frac{1}{2}$ and this amplitude ratio is given by $\frac{3}{2}$ it is a fair indication that kinetic growth trails in four dimensions are Gaussian (Fig. 2).

Next, we considered the probability $P_{N}$ of generation of trails of length $N$ (or larger). In Fig. 3 we plotted this quantity versus $1 / N$. $P_{N}$ does not decay to zero, but to a finite number $P_{\infty}$ relatively close to one, showing that a kinetic trail grows indefinitely with a finite probability $0.9932(1)$, i.e. only about one in 300 trails gets trapped. The partition function for open configurations, with the weights given by Eq. (2.6), is related to 


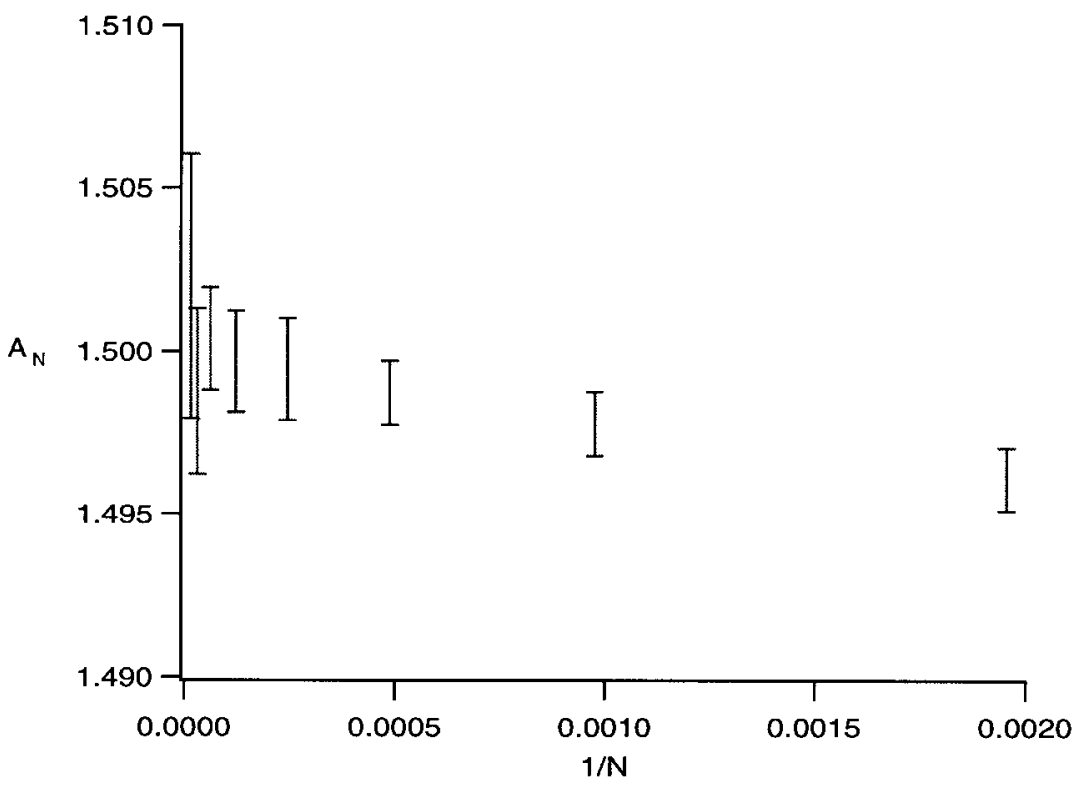

Fig. 2. The amplitude ratio $A_{N}$ is plotted versus $1 / N$.

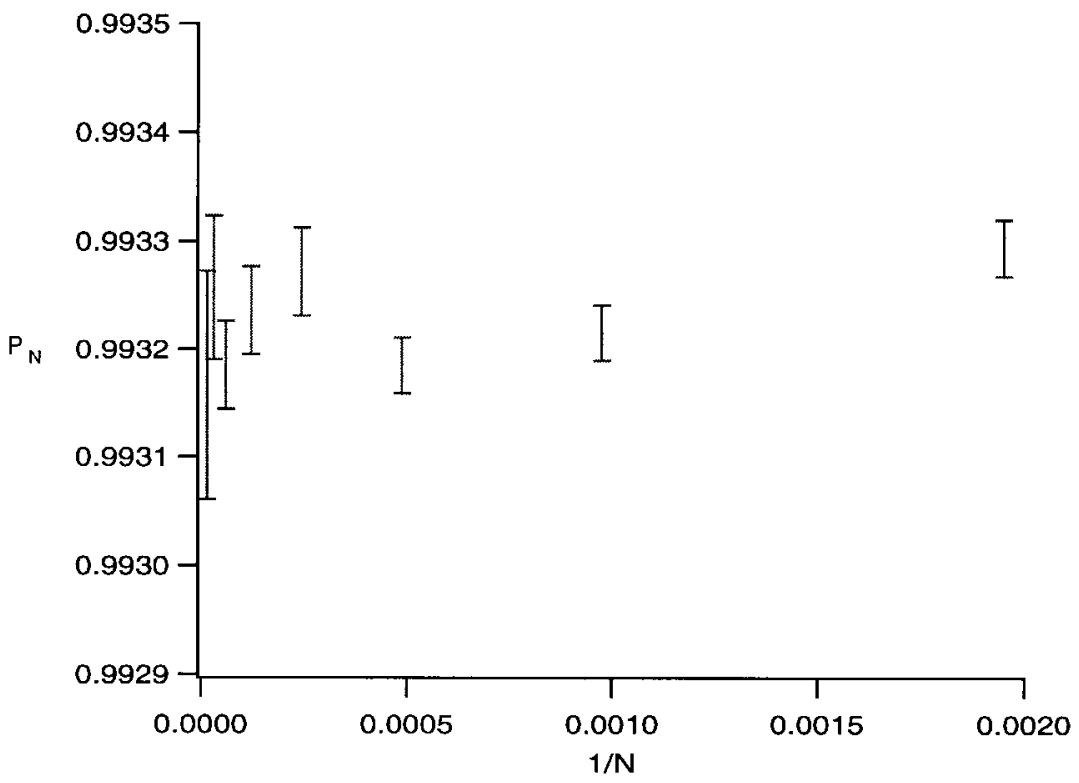

Fig. 3. The probability that a path has not yet been trapped, $P_{N}$, is plotted against $1 / N$. 


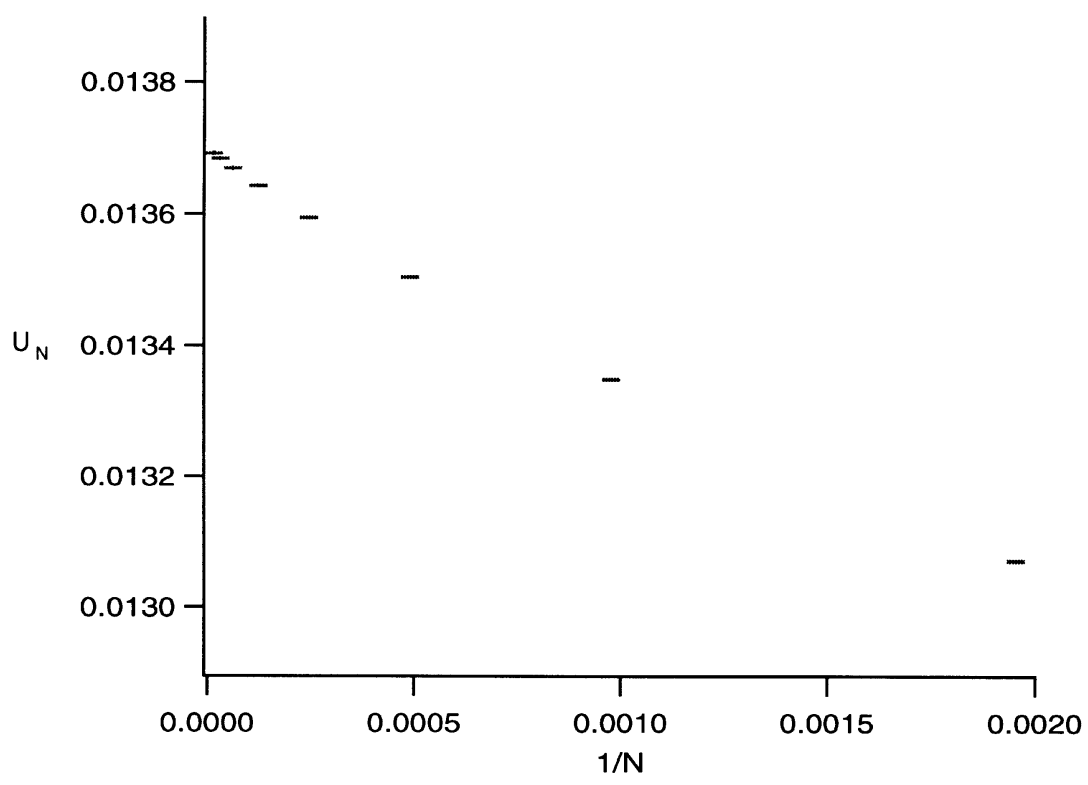

Fig. 4. The finite size internal energy $U_{N}$ is plotted against $1 / N$.

$P_{N}$ by

$$
Z_{N}=\sum_{\varphi_{N}} \omega_{2}^{m_{2}} \omega_{3}^{m_{3}} \omega_{4}^{m_{4}}=7^{N} \sum_{\varphi_{N}} p_{1}^{N-2 m_{2}-3 m_{3}-4 m_{4}} p_{2}^{m_{2}} p_{3}^{m_{3}} p_{4}^{m_{4}} \sim 7^{N} P_{N}
$$

where the $\sim$ is needed because the end-point contacts are not counted properly by the KGT. Since $P_{N} \rightarrow P_{\infty}>0$ this equation implies an exponent

$$
\gamma_{K G T}=1
$$

and the reduced free energy is equal to $\log (7)$. We were unable to adequately analyse the trapping rate

$$
Q_{N}=P_{N}-P_{N+1}
$$

which is equal to the probability of loop-formation.

The scaling of the internal energy $U_{N}$ is depicted in Fig. 4. The internal energy is a weighted combination of the mean number of 2-times, 3-times, and 4-times visited sites per step. We see a very slight curvature of the plot of $U_{N}$ versus $1 / N$. Thus, it is not possible to confidently extract a singular correction term of the form $N^{-\Delta_{u}}$, as it is not sufficiently different from a $1 / N$ correction. Of course even if one had estimated this correction exponent it is unclear whether this would allow one to infer the crossover or specific heat exponents. This is not only because of the possibility that the transition is first order but that the appropriate scaling relations [10] may not hold above the upper critical dimension. 


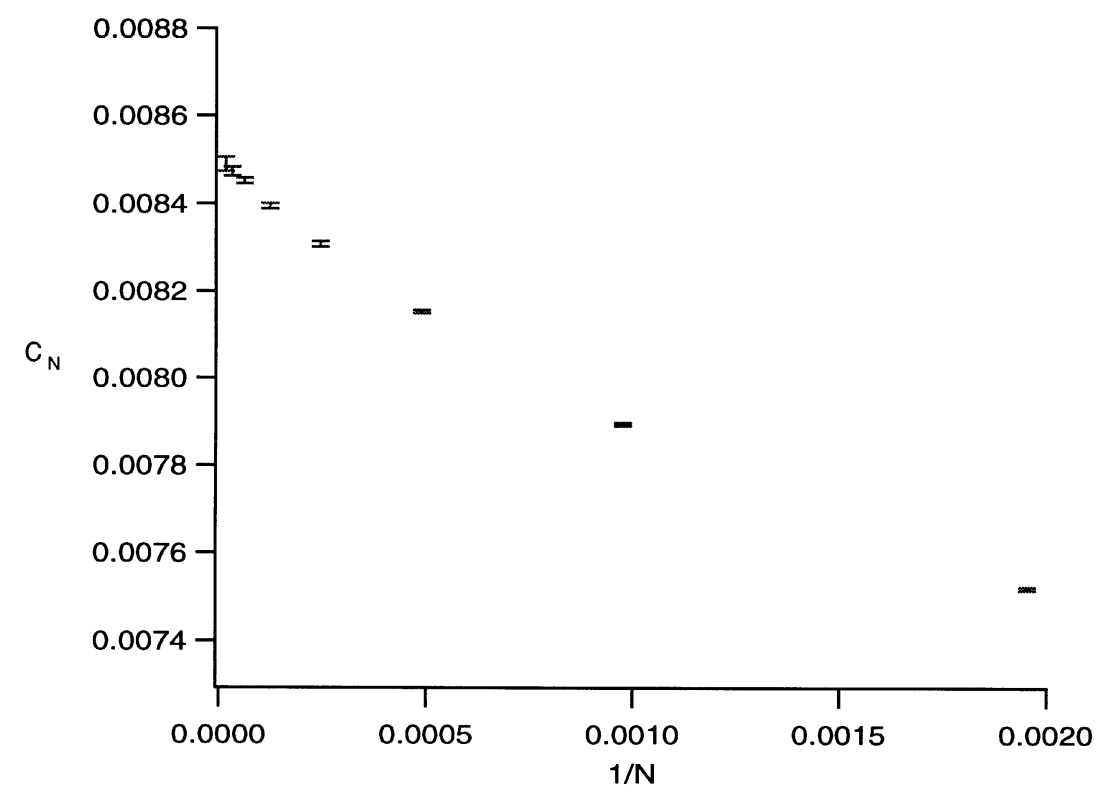

Fig. 5. The finite size specific heat $C_{N}$ is plotted versus $1 / N$.

In a similar manner, we have collected data for the specific heat $C_{N}$. As we see in Fig. 5, the specific heat converges, and there is a very slight curvature of the plot of $C_{N}$ versus $1 / N$, making any further analysis of singular behaviour difficult.

Our results certainly imply that the temperature given by the mapping from the kinetic model to the static one produces walks that are like random walks with respect to their geometric behaviour. The precision of the results for the internal energy and specific heat do not allow further analysis.

However, the results imply that there is a collapse transition in four dimensions and that the upper critical dimension is $d_{u}=3$. This concurs with the previous kinetic growth studies in lower dimensions.

\section{Conclusions}

We have conducted simulations of kinetic trails in four dimensions. Our kinetic model can be mapped to a static self-interacting model of trails. Given the working hypothesis, now tested in several dimensions and lattices, that kinetic growth trails at least identify the collapse temperature correctly, and perhaps also provide a description of the collapse point of the corresponding static problem, we have shown that there exists a collapse temperature in four dimension. This refutes a long standing hypothesis [11] that the collapse transition disappears above four dimensions. We have shown that the critical ISAT collapsing state is Gaussian, given that the usual scaling assumptions can be made. 


\section{Acknowledgements}

The authors take pleasure in thanking B. Drossel for carefully reading the manuscript. Financial support from the Australian Research Council is gratefully acknowledged by A.L.O. while both authors thank the Department of Physics at the University of Manchester where part of this work was completed. This work was supported by EPSRC Grant No. GR/K79307.

\section{References}

[1] P.-G. de Gennes, Scaling Concepts in Polymer Physics, Cornell University Press, Ithaca, 1979.

[2] B. Duplantier, H. Saleur, Phys. Rev. Lett. 59 (1987) 539.

[3] T. Prellberg, A.L. Owczarek, J. Phys. A 27 (1994) 1811.

[4] B. Duplantier, Europhys. Lett. 1 (1986) 491.

[5] B. Duplantier, J. Chem. Phys. 86 (1987) 4233.

[6] P.-G. de Gennes, J. Phys. Lett. 36 (1975) L55.

[7] M.J. Stephen, Phys. Lett. A 53 (1975) 363.

[8] B. Duplantier, J. Phys. 43 (1982) 991.

[9] J. des Cloizeaux, G. Jannink, Polymers in Solution, Clarendon Press, Oxford, 1990.

[10] R. Brak, A.L. Owczarek, T. Prellberg, J. Phys. A 26 (1993) 4565.

[11] M.A. Moore, J. Phys. A 10 (1976) 305.

[12] A. Malakis, Physica 84 (1976) 256.

[13] Y. Shapir, Y. Oono, J. Phys. A 17 (1984) L39.

[14] H.A. Lim, A. Guha, Y. Shapir, J. Phys. A 21 (1988) 773.

[15] I.S. Chang, A. Guha, H.A. Lim, Y. Shapir, J. Phys. A 21 (1988) L559.

[16] A.J. Guttmann, J. Phys. A 18 (1985) 567.

[17] A.J. Guttmann, J. Phys. A 18 (1985) 575.

[18] I. Guim, H.W.J. Blöte, T.W. Burkhardt, J. Phys. A 30 (1997) 413.

[19] I.S. Chang, H. Meirovitch, Phys. Rev. Lett. 69 (1992) 2232.

[20] A.L. Owczarek, T. Prellberg, J. Stat. Phys. 79 (1995) 951.

[21] T. Prellberg, A.L. Owczarek, Phys. Rev. E 51 (1995) 2142.

[22] P. Grassberger, R. Hegger, J. Phys. A 29 (1996) 279.

[23] D. Bennett-Wood et al., J. Phys. A 31 (1998) 4725.

[24] R. Brak, P.P. Nidras, A.L. Owczarek, J. Stat. Phys. 91 (1998) 75.

[25] A. Guha, H.A. Lim, Y. Shapir, J. Phys. A 21 (1988) 1043.

[26] H. Meirovitch, H.A. Lim, Phys. Rev. A 39 (1989) 4186.

[27] H. Meirovitch, H.A. Lim, J. Chem. Phys. 92 (1990) 5155.

[28] I.S. Chang, H. Meirovitch, Y. Shapir, Phys. Rev. A 41 (1990) 1808.

[29] J. Lyklema, J. Phys. A 18 (1985) L617.

[30] D. Knuth, The Art of Computer Programming, vol. 3: Sorting and Searching, Addison-Wesley, MA, 1969.

[31] N. Madras, G. Slade, The Self-Avoiding Walk, Birkhauser, Boston, 1993. 